\title{
Direct Detection of Low Molecular Weight Compounds in 2D and 3D Aptasensors by Biolayer Interferometry
}

Anthony Vignon ${ }^{\ddagger}$, Arthur Flaget ${ }^{\ddagger}$, Maxime Michelas, Mehdi Djeghdir, Eric Defrancq, Liliane

Coche-Guerente, Nicolas Spinelli,* Angéline Van der Heyden,* Jérôme Dejeu*

Univ. Grenoble Alpes, CNRS, DCM UMR-5250, F-38000 Grenoble, France

Corresponding authors: Nicolas Spinelli, + 334565208 33, nicolas.spinelli@univ-grenoblealpes.fr;

Angéline Van der Heyden, + 334565208 14, angeline.van-der-heyden@univ-grenoblealpes.fr

Jérôme Dejeu, + 334565208 13, jerome.dejeu@univ-grenoble-alpes.fr

Material and methods

Sensorgrams monitored upon incubation of L-Tym with a random DNA sequence immobilized on SA and SSA sensors

Sensorgrams monitored upon incubation of D-Tym with the $\mathrm{Apt}_{49}$ sequence immobilized on SA and SSA sensors S4

Residual plot for the fitted sensorgrams presented in Figure 1 ........................................... S5

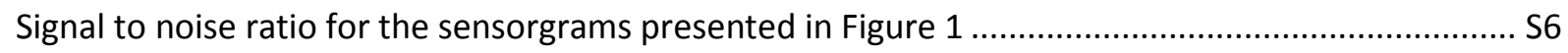

Limit of detection (LOD) and limit of quantitation (LOQ) for sensors presented in Figure 1 ............. S6

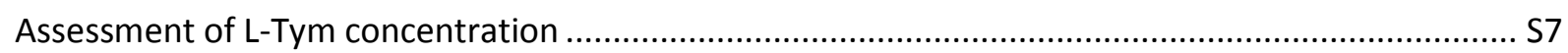

Comparison of SPR and BLI kinetic rate constants on 2D sensors ............................................. S8 


\section{Material and methods}

The recognition between Apt $_{49}$ and the L-tyrosinamide was analysed with an Octet Red 96 instrument using two types of sensors: streptavidin (SA) and super streptavidin (SSA) at $25^{\circ} \mathrm{C}$.

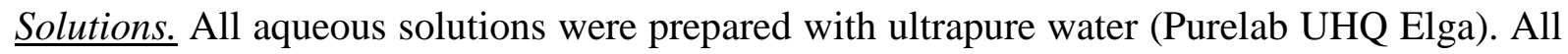
the chemical products ( $\mathrm{NaCl}, \mathrm{MgCl}_{2}$, Tween 20, Trizma base) were purchased from SigmaAldrich. Tris buffer (20 mM Trizma base, $50 \mathrm{mM} \mathrm{NaCl,} 5 \mathrm{mM} \mathrm{MgCl}_{2}$, Tween 20, 0.05\% v/v, pH 7.5 in ultrapure water) was used in each experiment. Oligonucleotides were dissolved in ultrapure water, aliquoted, evaporated and stored at $-20^{\circ} \mathrm{C}$. Before each measurement, DNA was dissolved in Tris buffer, heated at $90{ }^{\circ} \mathrm{C}$, and left to reach room temperature overnight.

Oligonucleotide Synthesis. The oligonucleotide sequences were prepared as previously described. ${ }^{1}$ Their sequences are the following: L-Tym aptamer (Apt $\left.{ }_{49}\right),{ }^{5}$ AAT TCG CTA GCT GGA GCT TGG ATT GAT GTG GTG TGT GAG TGC GGT GCC C $\mathrm{X}^{3^{\prime}}$ and random DNA sequence, ${ }^{5}$ TGA TCA GAT GAG CGT TCC CAG CAC TTC AGC CGA CGA TGC AAC CAG TTT $\mathrm{T} \mathrm{X}^{3^{\prime}}$, in which $\mathrm{X}$ represents biotin triethylene glycol (TEG) also named biotin-spacer 9. This spacer was introduced in order to decrease the steric hindrance that could be induced by the streptavidin layer.

Functionalization of sensors. BLI sensors, SA or SSA, were purchased from Forte Bio . Prior to use, they were immerged 10 minutes in buffer solution to dissolve the sucrose layer. Then the surfaces were dipped in an aptamer solution at $25 \mathrm{nM}$ or $100 \mathrm{nM}$ and rinsed in buffer solution during 10 minutes. The signal recorded at the end of the aptamer immobilization ( $\left.\Delta \lambda_{\text {aptamer }}\right)$ depends on both the dipping duration and the concentration of the aptamer. 
L-tyrosinamide recognition. The functionalized sensors were dipped in different Ltyrosinamide concentrations (5 only for SSA,10, 25, 50, 75, 100 and $150 \mu \mathrm{M}$ ) during at least 120 seconds interspersed by a rinsing step in the buffer solution during 2 minutes. Sensors with a Random DNA sequence were used as references to subtract the nonspecific adsorption.

Sensorgrams monitored upon incubation of L-Tym with a random DNA sequence immobilized on SA and SSA sensors

A

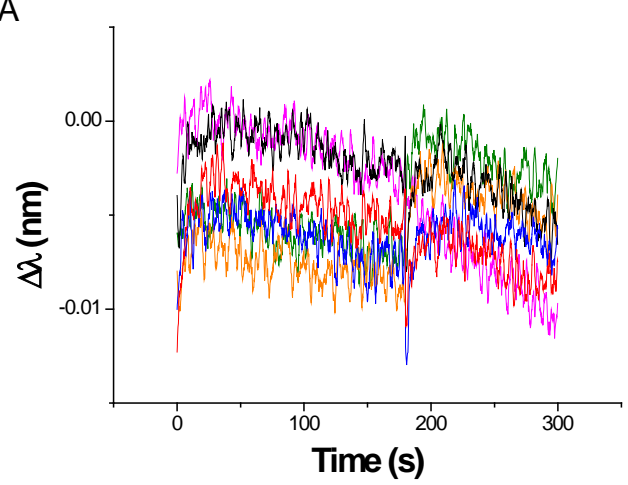

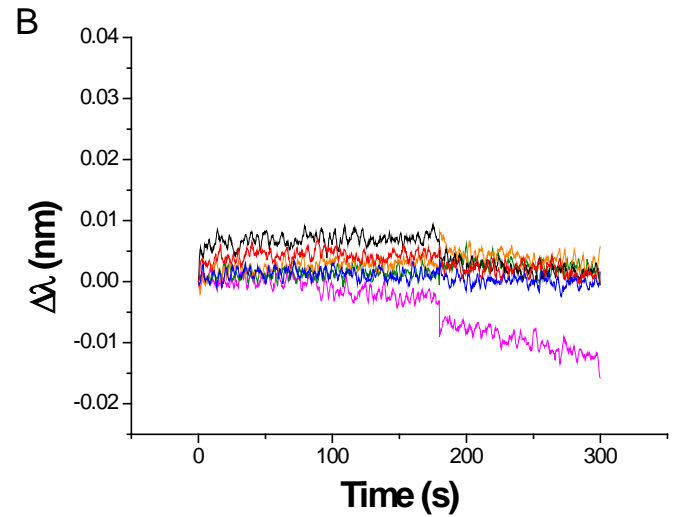

Figure S1: Sensorgrams recorded upon incubation of L-Tym at 5 (green), 10 (red), 25 (blue), 50 (pink), 100 (black) and $150 \mu \mathrm{M}$ (orange) on saturated sensors with a random DNA sequence on (A) SA and (B) SSA sensors 
Sensorgrams monitored upon incubation of D-Tym with the Apt 49 sequence immobilized on SA and SSA sensors

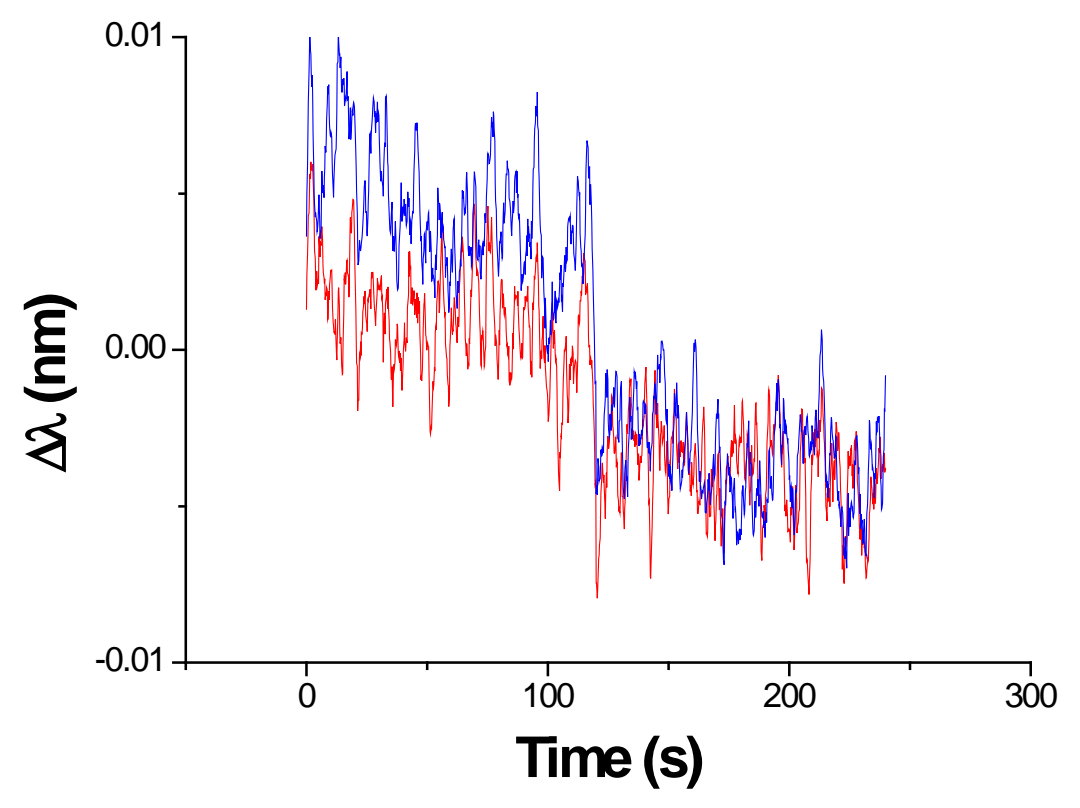

Figure S2: Sensorgrams recorded during incubation of D-Tym (1mM) with a saturating aptamer density surface (Apt 49 ) for SA (blue) and SSA (red) sensors. 
Residual plot for the fitted sensorgrams presented in Figure 1
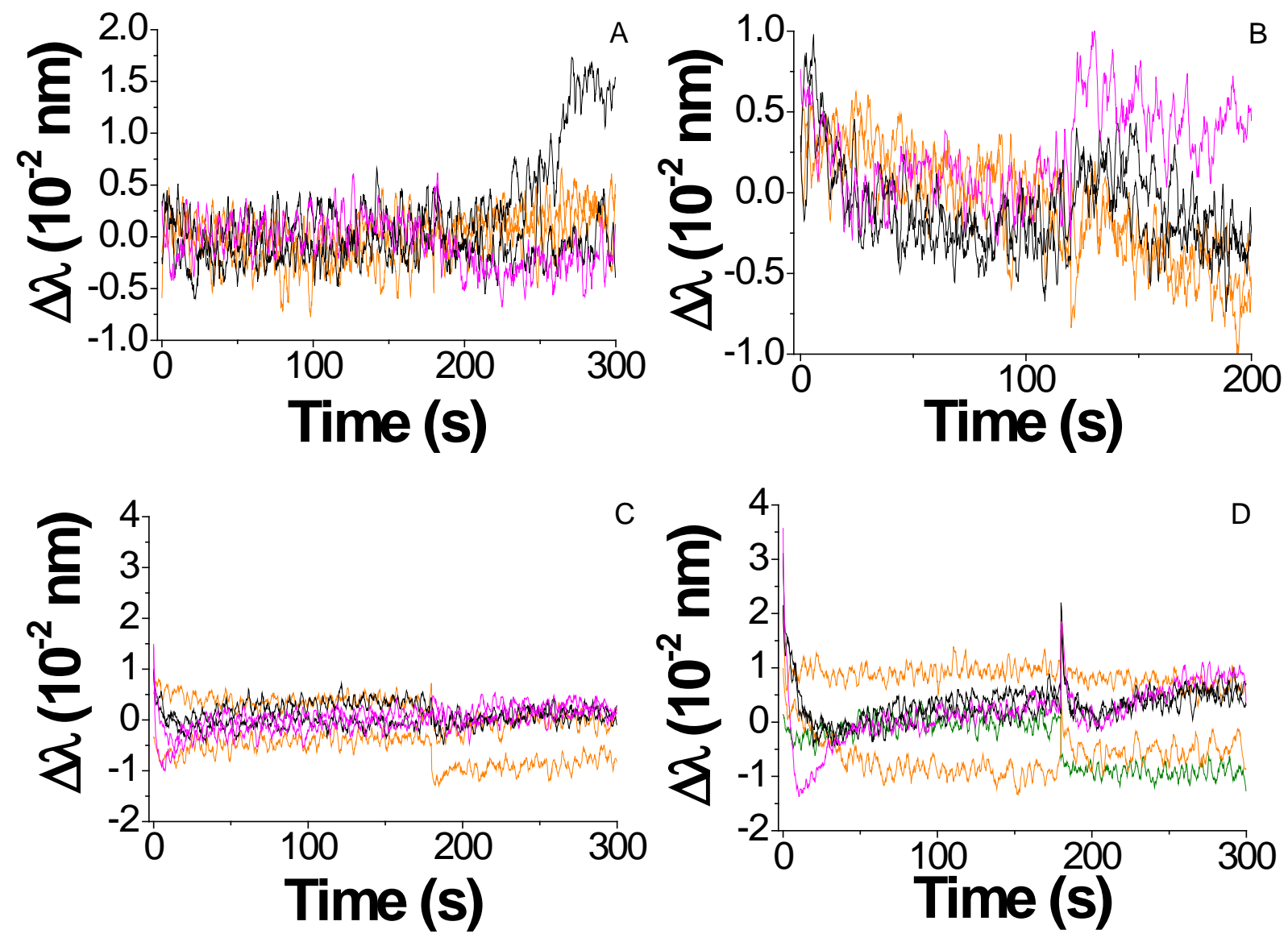

Figure S3: Residual plot for the fitted sensorgrams presented in figure 1 in the manuscript. (A) low aptamer density on SA; (B) high density of aptamer on SA; (C) low density of aptamer on SSA and (D) high density of aptamer on SSA. 
Signal to noise ratio for the sensorgrams presented in Figure 1

The signal-to-noise value given by the manufacturer is $3.5 \mathrm{pm}$ which is concordant with our experimental data (see below)
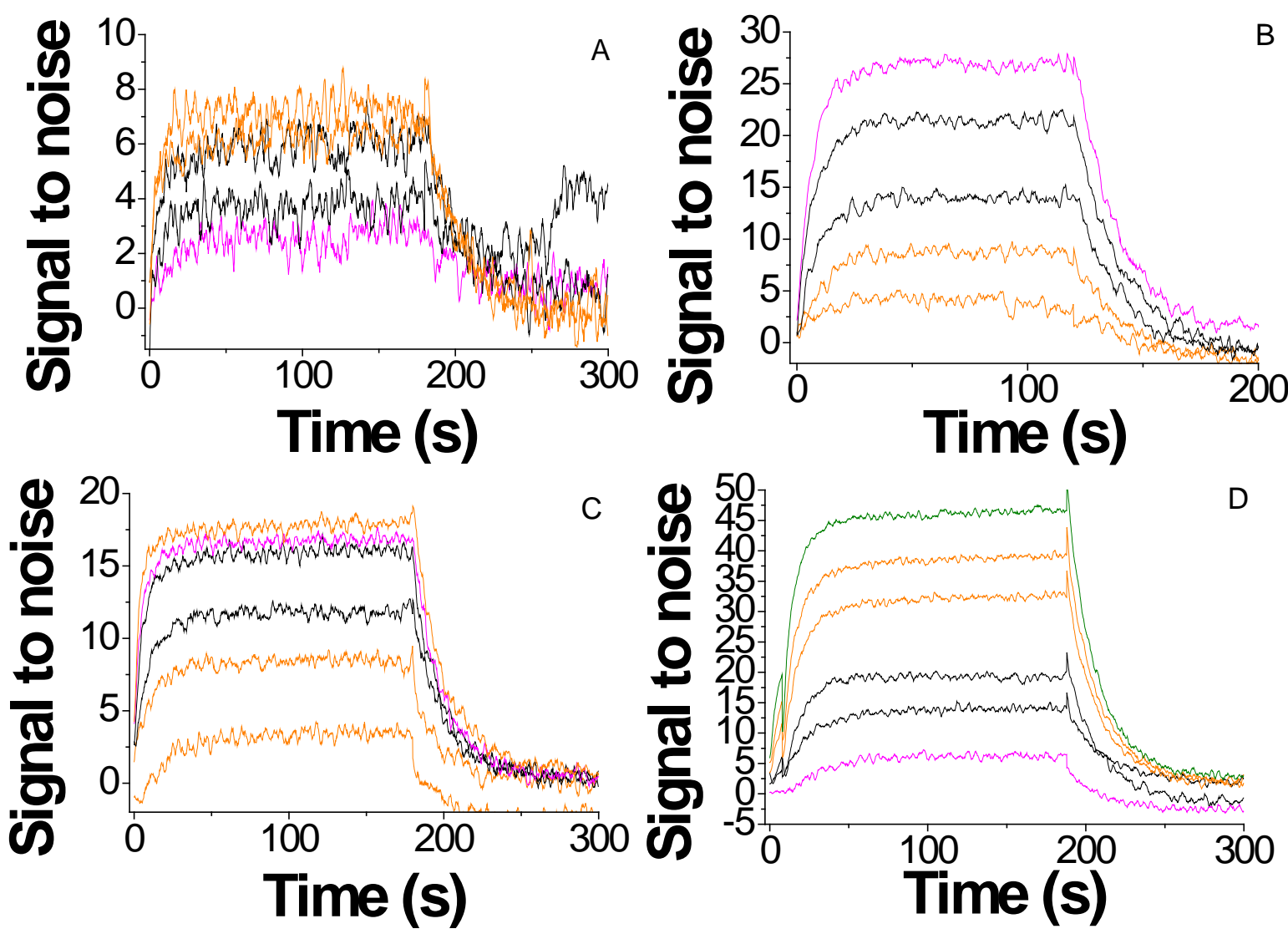

Figure S4: Signal-to-noise values for the sensorgrams presented in figure 1 in the manuscript. (A) low aptamer density on SA; (B) high density of aptamer on SA; (C) low density of aptamer on SSA and (D) high density of aptamer on SSA.

\section{Limit of detection (LOD) and limit of quantitation (LOQ) for sensors presented in Figure 1}

The limit of detection (LOD) and the limit of quantification (LOQ) were calculated with the following equations respectively:

$$
\begin{aligned}
& L O D=m_{\text {blank }}+3 \cdot S_{\text {blank }} \\
& L O D=m_{\text {blank }}+10 . S_{\text {blank }}
\end{aligned}
$$


Where $\mathrm{m}_{\text {blank }}$ and $\mathrm{S}_{\text {blank }}$ are the average and the standard deviation respectively of the blank measurements.

With the previous equation, LOD and LOQ values were calculated and are presented in table S1.

Table S1: Limit of detection (LOD) and limit of quantitation (LOQ) for the sensors presented in Figure 1.

\begin{tabular}{|c|c|c|c|c|c|c|c|}
\hline \multirow{2}{*}{$\begin{array}{c}\text { Sensor } \\
\text { type }\end{array}$} & \multirow{2}{*}{$\begin{array}{c}\text { Aptamer } \\
\text { density }\end{array}$} & \multirow{2}{*}{$\begin{array}{c}\mathrm{m}_{\text {blank }} \\
(\mathrm{nm})\end{array}$} & \multirow{2}{*}{$\begin{array}{c}\mathrm{S}_{\text {blank }} \\
(\mathrm{nm})\end{array}$} & \multicolumn{2}{|c|}{ LOD } & \multicolumn{2}{c|}{ LOQ } \\
\cline { 5 - 8 } & & & $\mathrm{nm}$ & $\mu \mathrm{M}$ & $\mathrm{nm}$ & $\mu \mathrm{M}$ \\
\hline \multirow{2}{*}{ SA } & Low & $7.3 .10^{-3}$ & $2.10^{-3}$ & 0.0133 & 15.9 & 0.027 & 394.1 \\
\cline { 2 - 8 } & high & $7.3 .10^{-3}$ & $2.10^{-3}$ & 0.0133 & 10.5 & 0.027 & 23.4 \\
\hline \multirow{2}{*}{ SSA } & Low & $3.4 .10^{-3}$ & $2.10^{-3}$ & 0.0094 & 2.8 & 0.023 & 8.7 \\
\cline { 2 - 8 } & high & $3.4 .10^{-3}$ & $2.10^{-3}$ & 0.0094 & 2.3 & 0.023 & 6.0 \\
\hline
\end{tabular}

Note that LOD and LOQ for SA sensors at low aptamer density were $16 \mu \mathrm{M}$ and $395 \mu \mathrm{M}$ respectively. These values rule out their possible use as sensor for L-Tym concentration assessment.

\section{Assessment of L-Tym concentration}

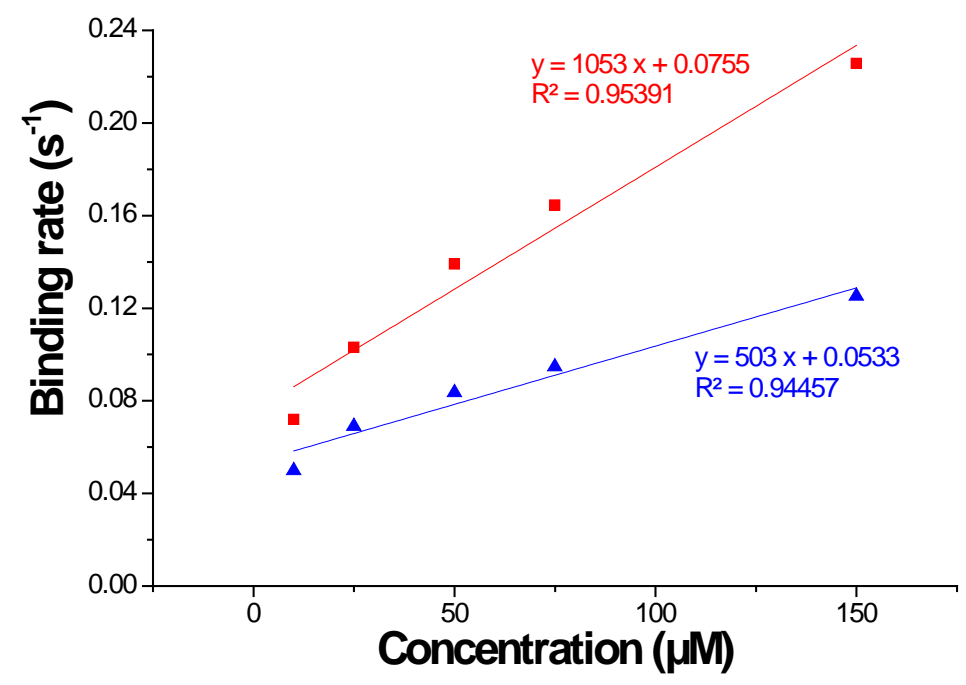

Figure S5: Standard curves for assessment of L-Tym concentration using SSA sensors with (red square) low and (blue triangle) high aptamer densities: Binding rate ( $\mathrm{k}_{\mathrm{obs}}$, obtained from sensorgrams fitting) versus L-Tym concentration in the incubation solution. 


\section{Comparison of SPR and BLI kinetic rate constants on 2D sensors}
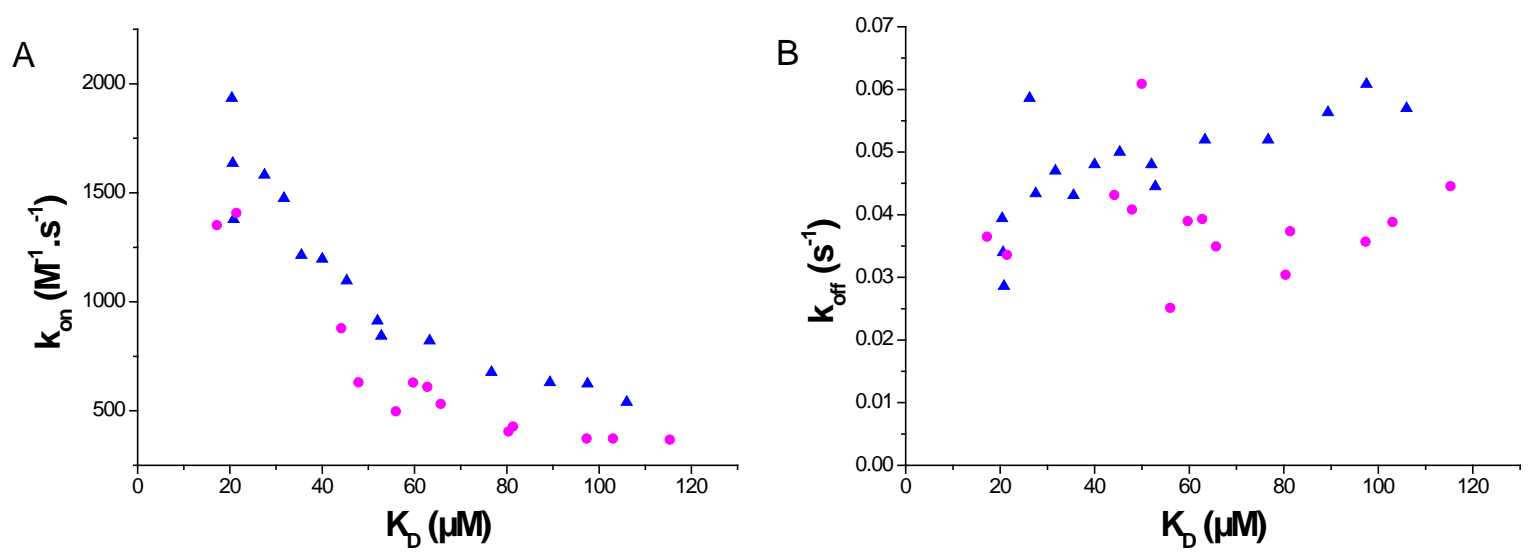

Figure S6: Variation of the kinetic rate constants: A) $k_{\text {on }}$ and B) $k_{\text {off }}$ for the BLI on SA sensors (Blue) and the $\mathrm{SPR}^{2}$ (pink) on a similar platform (2D aptamer layer) versus the equilibrium dissociation constant.

\section{References}

1. Osypova, A.; Thakar, D.; Dejeu, J.; Bonnet, H.; Van der Heyden, A.; Dubacheva, G. V.; Richter, R. P.; Defrancq, E.; Spinelli, N.; Coche-Guérente, L.; Labbé, P. Sensor Based on Aptamer Folding to Detect Low-Molecular Weight Analytes. Anal. Chem. 2015, 87, 7566-7574.

2. MacDonald, H.; Bonnet, H.; Van der Heyden, A.; Defrancq, E.; Spinelli, N.; CocheGuérente, L.; Dejeu, J. Influence of Aptamer Surface Coverage on Small Target Recognition: A SPR and QCM-D Comparative Study, J. Phys. Chem. C, 2019, 123, 13561-13568 